\title{
Perimeter intrusion detective system using arduino
}

\author{
Thiri Naing*1, Kyi Kyi Khaing ${ }^{2}$, Tin Tin Nwet ${ }^{3}$ \\ ${ }^{1}$ Department Information Technology Support and maintainance, University of Information Technology (UIT) \\ Yangon, +95- 9250376673 \\ ${ }^{2}$ Faculty of Computer System and Technology, Myanmar Institute of Information Technology (MIIT) \\ Mandalay, +95-9797367323 \\ *Corresponding author, e-mail: thuthiri@gmail.com¹, akyitaw07@gmail.com²,ttnwet@gmail.com³
}

\begin{abstract}
Perimeter intrusion detection systems are an integral part of most physical security systems. The main focus of the Perimeter Intrusion Detection System (PID) is on the fence intrusion. To secure public and private places such as military bases, airports, power station and security related application, the used of perimeter fencing is widely applicable for isolating and protecting. Fence structures merely prevent a percentage of intrusions or postpone them. Therefore, to monitor and investigate activities on or around the university, a higher level of security is needed. The system used major components as Arduino board, 8x8 LED display, ultrasonic sensor, 16x2 LCD display module and speaker. The unauthorized person who tries to intrude the university woull be sensed, detected and alarm would generate a signal that an intruder was trying to enter the university. The sound level depended on the distance, the nearer the intruder and stronger would be the alarm signal. PID system displayed the distance of the object or personal found in its region on the LCD display. This system was very useful for security applications. Ultrasonic sensor would be searching if there was a motion in its range.
\end{abstract}

Keywords: Aurdino UNO, 16x2 LCD, 8x8 LEDMatrix, HC-SR04 Ultrasonic Sensor, Speaker

Copyright $@ 2019$ APTIKOM - All rights reserved.

\section{Introduction}

The Internet of Things (IoT) has a great role in building today's smart environments. Along with those development based on the IoT model, security and privacy are two main issues need to be taken into consideration. Security threat can affect smart environment application in cause of the vulnerability in IoT-based system security. Therefore, designing intrusion detection systems (IDS) for IoT environments is a crucial aspect to alleviate security attacks toward the system. Conventional IDSs may not be an option for these matters since IoT devices have limited computing and storage capabilities with the specific protocol is being used [1].

An intruder detection system (IDS) on part of physical security as discussed in the paper, is an entry detector that sending an alarm on any entry or attempted entry by an intruder. It can be used for human surveillance by involving substitution of electronic surveillance device. IDS need to be designed professionally, planned, installed and maintenance regularly to make it affective and long-life. Unless these minimum standards are applied, the chance of an IDS becomes vulnerable to malfunction, circumvention and false alarms is high [2].

This field is actively researched, including the various alternative solution to solve the problems such as limited amount of energy and cost efficiency [3]. Home Automation is one of the most discussed topics in IoT. The idea to develop an inexpensive and safe system for indoor use has been an interesting researched area around the globe which plays a role in advances of technology and the presence of small, flexible and smart systems. The concepts of IoT was guiding us to develop an all-time surveillance using intelligent security system [4-7]. This paper proposes a university security system based on arduino with the use of HC-SR04 Ultrasonic Sensor and LCD display.

\section{Background Theory}

Security System has a set of Perimeter Intrusion Systems. PID system displays the distance of the object or personal found in its region on the LCD display. This system is very useful for security applications. Ultrasonic sensor will be searching if there is a motion in its range. 


\subsection{Hardware Requirement}

The basic components of an intrusion detection system are as follows:

- Sensors and their locations.

- Circuitry or communications infrastructure that connect sensors to a control unit and the control unit to an alarm display panel.

- A control unit that monitors the sensors, receives signals from them, and transmits an alarm signal.

- An alarm display panel with visual and/or sound enunciators that alert monitoring personnel to an intrusion.

Table 1 shows lists of hardware requirement.

Table 1. Lists of hardware requirement

\begin{tabular}{ccc}
\hline No. & Requirement & Quantity \\
\hline 1 & Arduino Board & 1 \\
2 & Ultrasonic Sensor & 1 \\
3 & $16 \times 2$ LCD Display & 1 \\
4 & $8 \times 8$ LED Matrix & 1 \\
5 & Speaker & 1 \\
6 & Breadboard & 1 \\
\hline
\end{tabular}

\subsubsection{Ultrasonic sensor module}

In this paper, HC-SR04 Ultrasonic sensor is used for sensor selection and is determined by the intrusion threat, the operating environment (e.g., indoors, outdoors), and power source constraints. To detect the presences of any person at the door, ultrasonic sensor HC-SR04 is being used here. The sensor module consists of ultrasonic transmitter, receiver and the control circuit. Ultrasonic Sensor consists of two circular eyes out of which one is used to transmit the ultrasonic wave and the other to receive it. Calculating the distance of the object based on the time taken by ultrasonic wave to return back to the sensor [9]. We can calculate the distance by the following formulae since the time and speed of sound is known.

\section{Distance $=($ Time $\mathrm{x}$ Speed of Sound $) / 2$}

See the following project to gauge the distance of any object and to grasp how the Ultrasonic sensor is working. Figure 1 shows HC-SR04 Ultrasonic sensor and Table 2 shows it range defining diagram described the following. Figure 2 shows sensor range defining diagram.

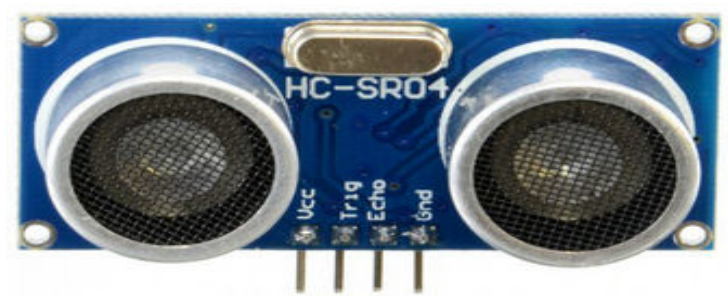

Figure 1. HC-SR04 ultrasonic sensor

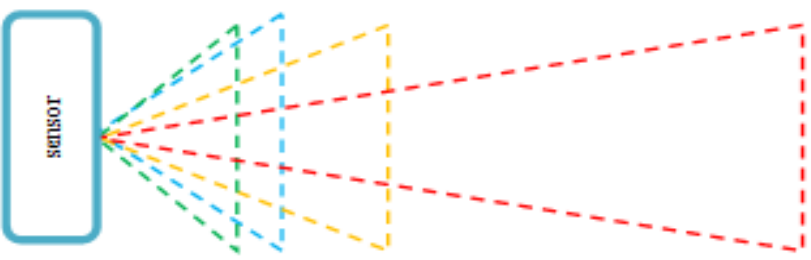

Figure 2. Sensor range defining diagram 
Table 2. Range defining for $8 \times 8$ matrix

\begin{tabular}{ccc}
\hline No. & Square & Range \\
\hline 1 & $2 \times 2$ Square & $2 \mathrm{~cm}-30 \mathrm{~cm}$ \\
2 & $4 \times 4$ Square & $31 \mathrm{~cm}-60 \mathrm{~cm}$ \\
3 & $6 \times 6$ Square & $61 \mathrm{~cm}-120 \mathrm{~cm}$ \\
4 & $8 \times 8$ Square & $121 \mathrm{~cm}-300 \mathrm{~cm}$ \\
\hline
\end{tabular}

\subsubsection{Arduino board}

Arduino Uno is a microcontroller board based on the ATmega328P. It has 14 digital input/output pins (of which 6 can be used as PWM outputs), 6 analog inputs, a $16 \mathrm{MHz}$ quartz crystal, a USB connection, a power jack, an ICSP header and a reset button. Figure 3 shows the Arduino board.

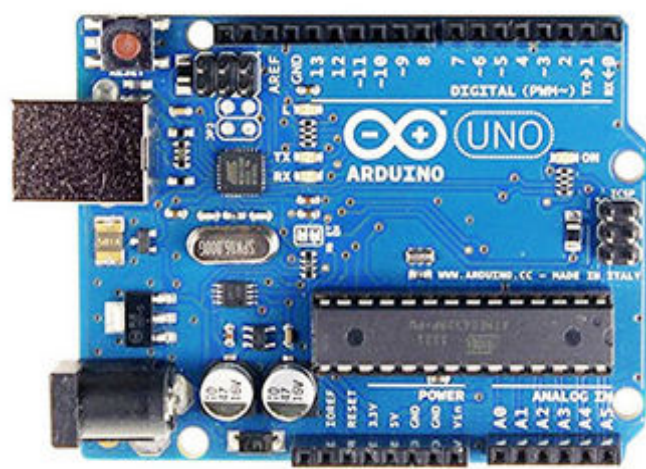

Figure 3. Arduino UNO board

\subsubsection{Pin connection with components and arduino board}

Table 3 shows the pin connection with components and Arduino board.

Table 3. Pin connection with components and arduino board

\begin{tabular}{llll}
\hline No. & Pin No. of Arduino & Pin of Components & Components \\
\hline 1 & 2 & RS & \\
2 & 3 & E & \\
3 & 4 & D4 & $16 \times 2$ LCD Display \\
4 & 5 & D5 & \\
5 & 6 & D6 & \\
6 & 7 & D7 & $8 \times 8$ Matrix \\
7 & 8 & CLK & \\
8 & 9 & CS & Ultrasonic Sensor \\
9 & 10 & DIN & Speaker \\
10 & 11 & Trig & \\
11 & 12 & Echo & Speaker \\
12 & 13 & & \\
\hline
\end{tabular}

Pins of LCD are connected with Arduino in this way $\mathrm{RS}=2$, En = 3, D4 = 4, D5 = 5, D6 = 6, D7 $=7$. Pins of $8 \times 8$ dot matrix (MAX7219) are connected with Arduino in this way.

$\mathrm{CLK}=8$

$\mathrm{CS}=9$

$\mathrm{DIN}=10$

Pins of ultrasonic range detector are connected with Arduino in this way.

echoPin $=12$;

trig Pin $=10$; 
Pin of speaker is connected with Arduino pin13. And then ground and Vec will be given to the circuit as shown in Figure 4. This circuit will detect objects between its detection range. The speaker will generate sound after one complete loop when the object is in the nearest range (L4 $=0 \mathrm{~cm}$ to $10 \mathrm{~cm}$ ). The speaker will generate sound after three complete loops when the object is in second nearest range $(\mathrm{L} 3=10 \mathrm{~cm}$ to $20 \mathrm{~cm})$. The speaker will generate sound after six complete loops when the object is in third nearest range $(\mathrm{L} 2=20 \mathrm{~cm}$ to $30 \mathrm{~cm})$. The speaker will generate sound after nine complete loops when the object is away starting from fourth nearest range ( $\mathrm{L} 1=40 \mathrm{~cm}$ to away). Figure 5 depicts the Block diagram of PID system

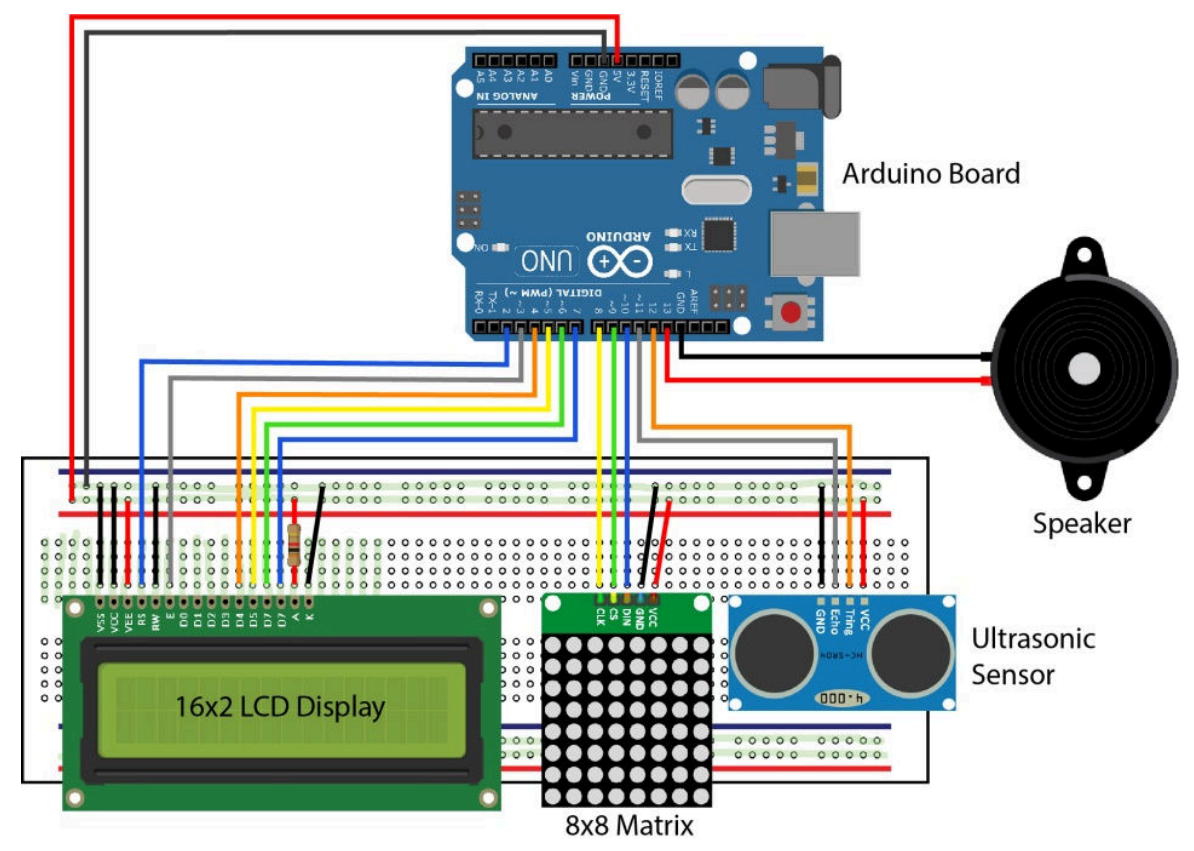

Figure 4. Circuit diagram of PID system

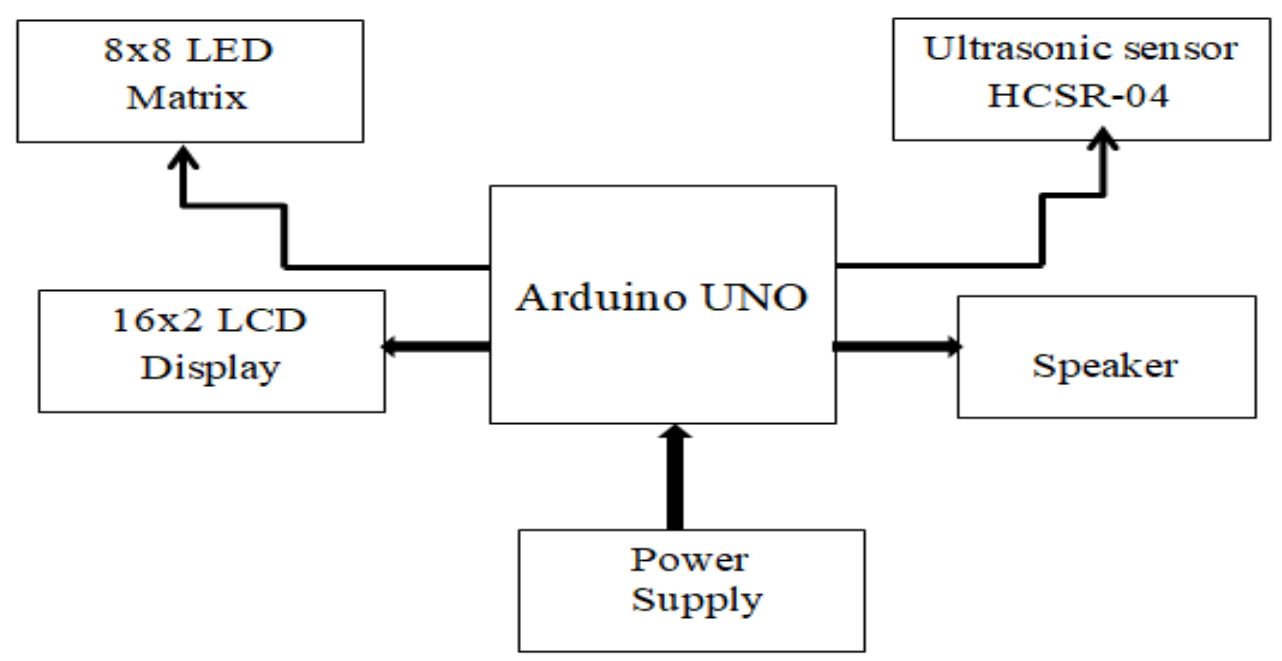

Figure 5. Block diagram of PID system 


\subsubsection{Basic 8x8 LED matrix working system}

If the sensor detects something gets closer to the sensor, it will send a feedback to the processing unit. LCD display will display the distance of the intruder. In Figure 6, 8x8 LED display will show 4 stages. The alarm will ring with increasing volume as the object coming closer [8].

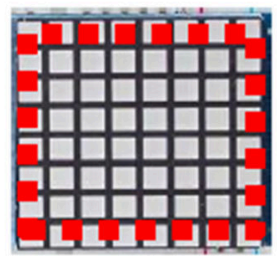

Blinking

No Intrusion

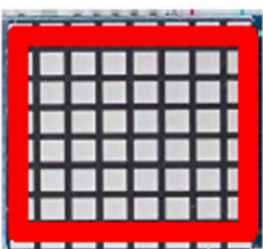

Intruder Found!!

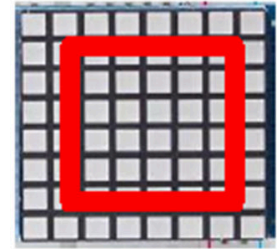

Intruder coming close !!

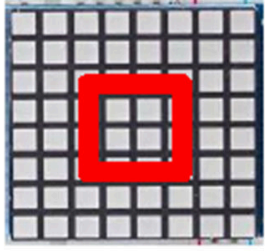

Intruder near !!

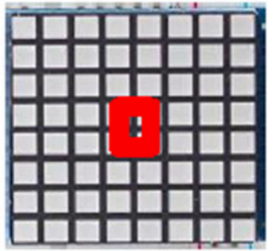

Intruder is too close!!

Figure 6. Four stages on 8x8 LED display

\section{System Operation}

When an object comes in the range of the motion sensor, the Arduino is interrupted by the matrix and the Arduino sends signal to 8x8 LED matrix display and lights up all LEDs. When an object comes in the range of the ultrasonic sensor, the Arduino calculates the distance to that object. Then it sends it to LCD to display the distance and a particular message. The distance is mapped into 4 ranges to display it on 8x8 LED matrix display. When there is no object, the outermost LEDs blink. As the object comes closer, the LEDs will become solid row without blinking. When the object comes too close to the sensor, the innermost LEDs will blink. The distance is mapped into a variable in the range ' 1 ' to ' 10 ' while ' 10 ' being the furthest and ' 1 ' being the nearest. When the object is near, the Arduino do some processing and determines which frequency to make for the speaker, and the speaker makes sound. The further the object, the lower the delay between each frequency. Therefore, it sounds like the frequency changes. Then the cycle is repeated.

\subsection{Software Procedure of the system}

Figure 7 shows four separate different condition for displaying $8 \times 8$ matrix display. If the intruder I in the range between 2 and 30 centimeters, $8 \times 8$ matrix display will show dot2Square.

- If in the range of $60 \mathrm{~cm}, 8 \times 8$ display will show d0t4Square.

- If in the range of $120 \mathrm{~cm}, 8 \times 8$ display will show dot6Square.

- If in the range greater than $120 \mathrm{~cm}, 8 \times 8$ matrix displays will show dot8Square matrix which means nothing is approaching towards the center.

We test that maximum range of our Ultrasonic sensor estimated is $300 \mathrm{~cm}$ and angular coverage is around 15 degree. For measuring the distance between sensor and object function.

Declaring variable "duration" for assigning the value of the time travelled by the ultrasound between sensor and the object. Library function "pulseIn" is used to trigger the sensor to emit ultrasound. We get the value of time travelled by the ultrasound between sensor and the object by this method. If we want the distance of the object, we have to multiply by the velocity of sound which is 34300 centimeters per second and 1.3503 inches per second. In this case, we have to divide the velocity by 2 in order to have the value for velocity of echoing sound wave.

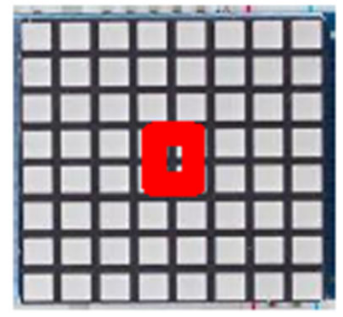

(a)

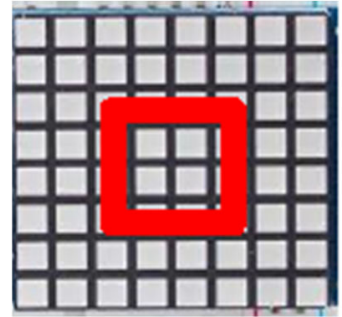

(b)

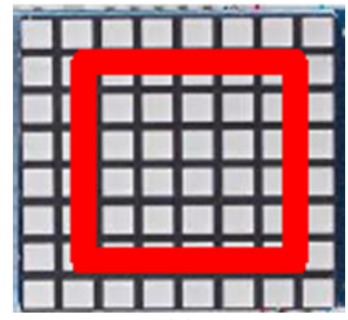

(c)

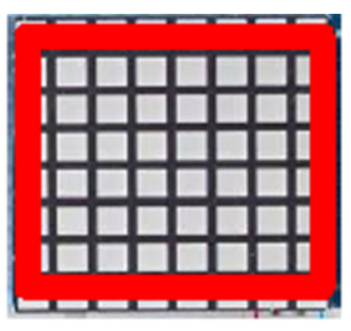

(d)

Figure 7. 8x8 matrix display, (a). dot2Square, (b). dot4Square, (c). dot6Square, (d). dot8Square 
After hard working, all the parts are connected as circuit design. Then we upload the programming code as we compiled in the Arduino and we get positive result. At last we see that it works properly according to our design. Figure 8 describes the intruder is found and very close to the campus.

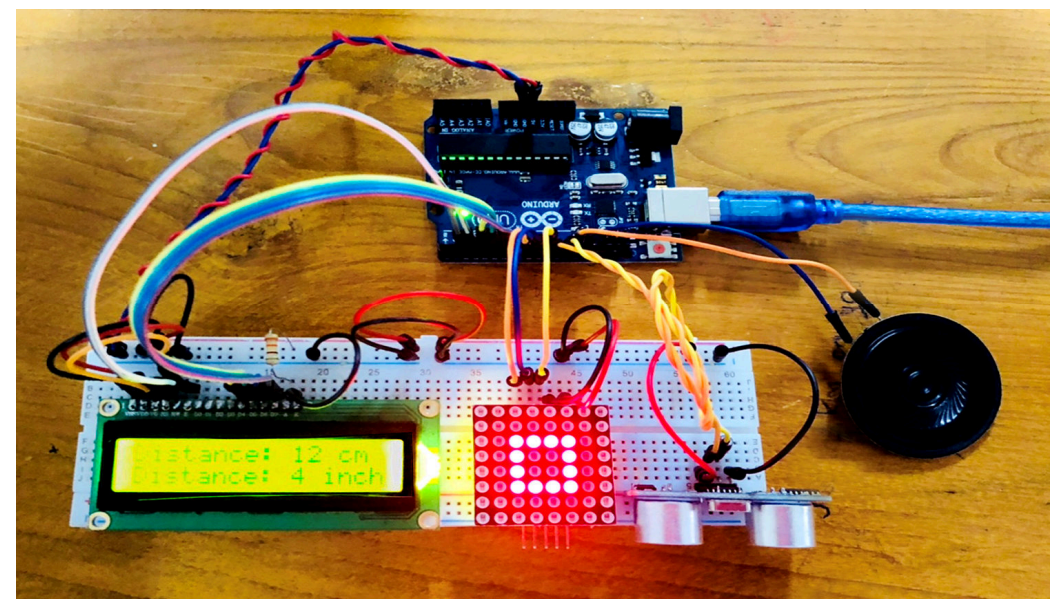

Figure 8. Output display of PID system

\section{Conclusion}

The arduino-based perimeter intrusion detection (PID) system by using Ultrasonic sensor (HCSR-04) is designed by both hardware and software controlled. Places like Bank, Office, University, and Home are in need of security for the aim of safety including to avoid intrusion. PID system is highly preferable to tackle those matters. The system develops a novel approach that automatically detects intruder and produces a warning alarm, display on the LCD. We found that the maximum loudness of our speaker is about $4000 \mathrm{~Hz}$. So, we define that from $2000 \mathrm{~Hz}$ of sound will alarm when the intruder is at the outermost range to $4000 \mathrm{~Hz}$ of alarm song will ring when the intruder is nearest of the sensor. So, if the object is coming near, sound of alarm increase volume and delay time of alarm will be lesser or alarm will ring faster.

\section{References}

[1] https://journalofcloudcomputing.springeropen.com

[2] https://www.sciencedirect.com/topics/computer-science/intruder-detection

[3] F. Samie, L. Bauer, and J. Henkel. IoT Technologies for Embedded Computing: A Survey, in CODES+ISSS, 2016.

[4] L. Ophir, 802.11 over Coax - A Hybrid Coax - Wireless Home Network Using 802.11 Technology. Consumer Communications and Networking Conference. Jan. 2004: 13-18.

[5] B. Yuksekkaya, A.A. Kayalar, M.B. Tonsun, M.K.Ozean, and A.Z. Alkar, A GSM, Internet, and Speech Controlled Wireless Interactive Home Automation System, IEEE Transactions on Consumer Electronics, 2006; 52(3): 837-843.

[6] M. Khan, B. N. Silva, K. Han, Internet of Things based energy-aware smart home control system, IEEE Access, 2016: 4: 7556-7566.

[7] Sivanathan A., Sherratt D., Gharakheili H., Sivaraman V., Vishwanath A., Low-cost flow-based security solutions for smart-home IoT devices," 2016 IEEE International Conference on Advanced Networks and Telecommunications Systems (ANTS). 2016.

[8] https://www.instructables.com/id/8x8-LED-Matrix-Using-Arduino/

[9] What is an Ultrasonic Sensor?, https://www.keyence.com/ss/products/sensor/sensorbasics/ultrasonic/info/

[10] https://randomnerdtutorials.com/complete-guide-for-ultrasonic-sensor-hc-sr04/ 